\title{
Post Colonoscopy Splenic Injuries: Case Series and Recommendations
}

\author{
Hassan Malik $^{\mathrm{a}, \mathrm{b}}$, Drago Popovic ${ }^{\mathrm{a}}$, Henk Van Rooyen ${ }^{\mathrm{a}}$
}

\begin{abstract}
Splenic injury is an uncommon, but serious complication of colonoscopy. Any cause of excessive spleno-colic adhesions, splenomegaly, or underlying splenic disease would be a predisposing factor for splenic injury during colonoscopy. A delay in diagnosis has been noted as many physicians are not aware of this complication of colonoscopy. We report two cases of splenic injury secondary to screening colonoscopy. The first refers to a 61-year-old male, who was involved in a motor vehicle accident a month prior to the procedure; with the second case that of a 46-year-old healthy female. Clinical, biochemical and radiological findings were suggestive of a splenic rupture and hemoperitoneum. Both patients had grade III splenic ruptures with hemodynamic instability, which warranted urgent splenectomy. Both patients had an uneventful recovery. Proposed mechanisms are traction on the spleno-colic ligament leading to splenic capsule avulsion or direct splenic trauma due to colonoscopic manipulation. So-called "looping of the colonoscope" in the bowel, followed by aggressive straightening can cause strain on the spleno-colic ligament and tearing of blood vessels. The presence of Kehr's sign in an hemodynamically unstable patient is a very sensitive pointer towards splenic rupture. Patients with recent abdominal trauma should be properly screened with appropriate history and investigations prior to colonoscopy. CT scan of the abdomen is the test of choice for the evaluation of splenic injury. Splenectomy is the most commonly performed procedure for post-colonoscopy injuries of the spleen.
\end{abstract}

Keywords: Splenic injury/grade; Colonoscopy; Splenectomy; Kehr's sign

\section{Introduction}

Colonoscopy is a commonly performed procedure for both

\footnotetext{
Manuscript accepted for publication September 11, 2013

${ }^{a}$ Hervey Bay Hospital, Uraween Road, Queensland, Australia

${ }^{\mathrm{b}}$ Corresponding author: Hassan Malik, Hervey Bay Hospital, Uraween

Road, Queensland, Australia. Email: hazymalik@yahoo.com
}

doi: http://dx.doi.org/10.4021/jmc1498w diagnostic and therapeutic purposes. It is an effective minimally invasive way to examine and sample tissue from the gastrointestinal tract. It has a low rate of complications $[1$, 2], with hemorrhage ( $1.8-2.5$ percent) and perforation ( 0.34 - 2.14 percent) being the most common. These are more prevalent with therapeutic procedures such as polypectomy or dilatation [3, 4]. Extra-colonic or visceral complications include pneumothorax, pneumomediastinum, mesenteric injury and colonic volvulus $[5,6]$.

Splenic rupture is an uncommon but potentially lethal complication. Not much documentation is found in the medical literature. For this publication a PubMed search was performed. It was reported by Wherry and Zhener in 1974 and so far less than 100 cases have been published [7, 8]. However, with the increasing use of colonoscopy endoscopists, surgeons and radiologist will more often encounter this complication.

The most likely mechanism of injury is that of traction on the spleen by way of pre-existing adhesions [9]. This occurs during manipulation of the colon at the time of colonoscopy. Excessive spleno-colic adhesions, splenomegaly and other splenic pathology are therefore regarded as predisposing factors. Patients with a history of recent abdominal trauma are also at risk, as will be discussed below.

A delay in diagnosis has been noted in the literature, the main reason being the ignorance of many physicians regarding this potential negative outcome. Splenic rupture should

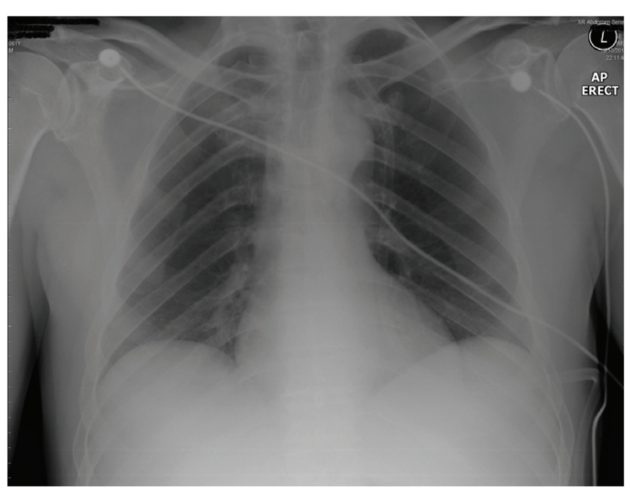

Figure 1. Unremarkable chest $X$ ray. 


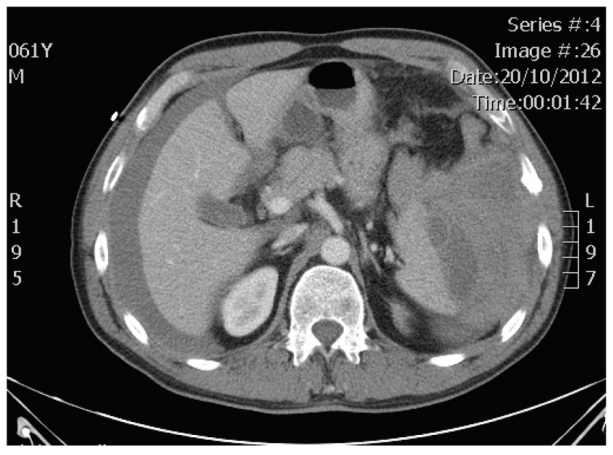

Figure 2. Hemoperitoneum with splenic hematoma.

be considered in all presentations of abdominal pain and hemodynamic instability after colonoscopy, especially with a history of past surgical interventions and therefore potential spleno-colic adhesions. Patients with recent abdominal trauma should be screened with appropriate investigation beforehand.

We present two cases of splenic injury secondary to colonoscopy. The first is a 61-year-old male that was involved in a high speed motor vehicle accident one month prior to colonoscopy. The second is a 45 -year-old female with no significant past medical history. Both presented with abdominal pain and hypovolemic shock, a few hours after colonoscopy. Both warranted splenectomy as a lifesaving procedure.

\section{Case Report}

\section{Case 1}

A fit 61-year-old male without significant medical history

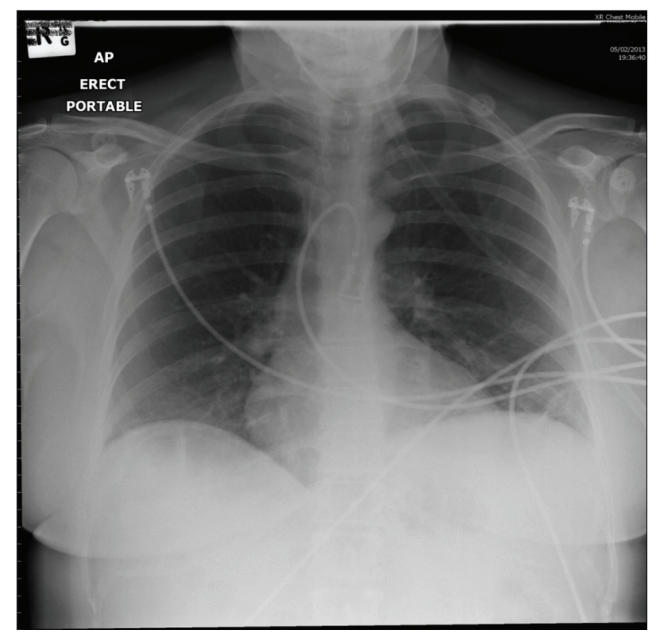

Figure 3. CXR showing no free air under the diaphragms.

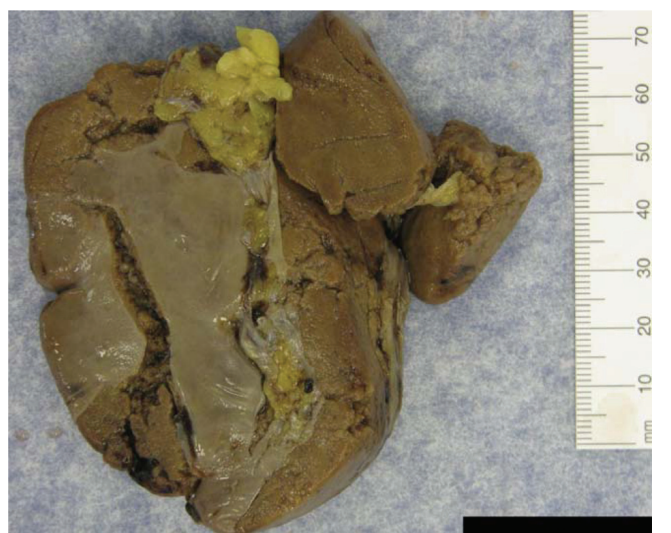

Figure 4. Full thickness splenic laceration with capsule stripping.

underwent colonoscopy as regular surveillance for a family history of bowel cancer. He had been involved in a high speed motor vehicle accident one month earlier, when his car had skid off the road into a tree. He self extricated, and did not suffer any major injuries. Paramedical staff attended to him and discharged him home in a stable condition. Persistent left sided chest pain was treated with simple analgesics.

The colonoscopy was uneventful, without difficulty, well tolerated and he was discharged home two hours postprocedure. Four hours post colonoscopy he developed abdominal pain, felt light headed and collapsed at home.

On admission to emergency department he was hypotensive (blood pressure 92/62, Hemoglobin $106 \mathrm{~g} / \mathrm{L}$ ). He complained of pain in the left shoulder (Kehr's sign), had generalized abdominal pain and was acidotic (serum $\mathrm{pH}$ 7.2, lactate $3.43 \mathrm{mmol} / \mathrm{L}$ ). Digital rectal examination and chest

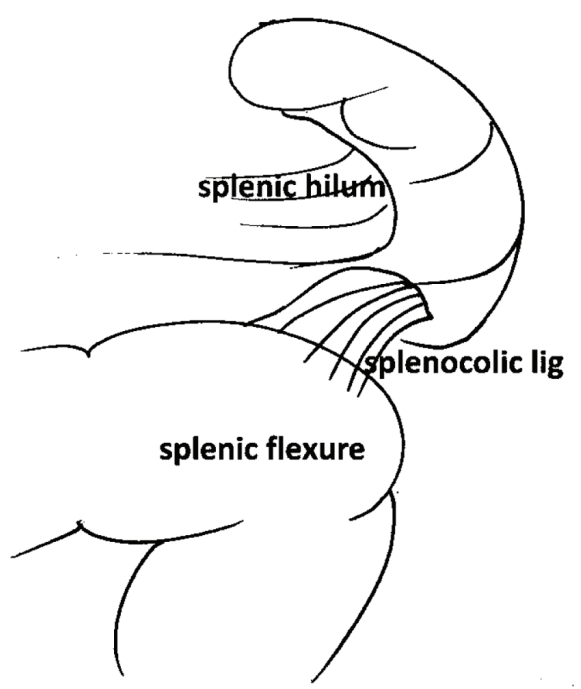

Figure 5. Excessive downward stretching of the splenocolic ligament can induce a tear in the splenic capsule and severe hemorrhage (courtesy of Dr Drago Popovic, Hervey Bay Hospital, Australia). 
X-ray were normal (Fig. 1). Resuscitation commenced with intravenous fluids and four units of packed red blood cells transfused. CT scan of the abdomen revealed a Grade III splenic hematoma with associated hemoperitoneum (Fig. 2).

The patient underwent emergency laparotomy, at which 4.5 Litres of blood was removed from the abdominal cavity. A ruptured subscapular hematoma was present at the lower pole of the spleen. A splenectomy was performed, delivering a 91 gram spleen with histologically confirmed rupture [10]. The patient made an uneventful recovery.

\section{Case 2}

A healthy 46-year-old female presented with left upper quadrant abdominal pain, radiating to the left shoulder. Six hours earlier, she had a colonoscopy for investigation of rectal bleeding and had a sigmoid polypectomy. On presentation the patient was hemodynamically unstable with pallor, diaphoresis, hypotension (blood pressure 84/58) and anaemia (Hemoglobin $107 \mathrm{~g} / \mathrm{L}$ ). Examination further revealed significant generalized abdominal tenderness with guarding in the left upper quadrant and a positive Kehr's sign. A chest $\mathrm{X}$-ray was normal without evidence of free intra-peritoneal air (Fig. 3).

Despite active resuscitation with 2 litres of saline, the patient remained hypotensive and developed tachycardia (pulse 120 per minute). Due to her unstable condition and inability to lie supine due to severe pain, $\mathrm{CT}$ abdomen was deferred. The patient had a diagnostic laparoscopy with follow on exploratory laparotomy, all of these performed within 3 hours of her initial presentation. An hemoperitoneum with clot formation in the left upper quadrant was encountered; this was due to a laceration of the lower half of the spleen leading to eventual splenectomy. Apart from 2 litres of blood loss, no other significant findings were encountered. She required 5 units of packed red blood cells to maintain end organ and tissue perfusion. She was discharged home seven days post-operative with a stable hemoglobin. The histology revealed a full thickness $4.5 \mathrm{~cm}$ laceration in an 88.8 gram spleen, consistent with a Grade III splenic injury (Fig. 4).

\section{Discussion}

No definitive pathophysiology has been established to explain the reason for splenic injury following colonoscopy. One proposed mechanism referred to traction on the splenocolic ligament (or previously formed splenocolic adhesions) leading to an avulsion related injury of the spleen. Another mechanism could be direct contact splenic trauma due to colonoscopic manipulation within the bowel $[9,11]$. The splenocolic adhesions are mostly found between the splenic angle of the colon and the spleen. It is suspected that these adhesions are stretched by the manipulation of the colon.
Such can be "looping of the colonoscope" in the colon, a simple rotational manoeuvre to guide the colonoscope past the splenic and hepatic flexure, followed by gentle straightening of the scope. A forceful straightening may increase the tension on the splenocolic ligament, leading to tearing of blood vessels in the vicinity of inferior polar artery, without necessarily the ligament itself (Fig. 5).

Patients with previous abdominal surgery are at a proposed increased risk for splenic injury due to the possibility of splenocolic adhesions. This is more commonly encountered in females. A review by Saad et al gives the risk ratio for females to males as 3.2:1 [12].

Another important consideration is the past history of trauma in patients due for colonoscopy. In our first case report, citing a patient with abdominal trauma a short while before the procedure, a subacute subcapsular splenic hematoma could have been present, thus leading to splenic capsular rupture with minimal colonoscopic manipulation. A history of recent trauma should hence be considered a contributing factor to splenic rupture in post-colonoscopy patients and needs to be elicited from the history.

The presence of Kehr's sign in a hemodynamically unstable patient is a very important indicator of splenic rupture. Nausea, vomiting with hypovolemia and sudden onset of anaemia post-colonoscopy is often associated with splenic ruptures. With these clinical signs and in the absence of visible hemorrhage per rectum, a splenic injury should be suspected and therefore treated as an emergency. Review of the literature indicates that most of these signs do appear within the first 24 hours following the procedure.

A contrast enhanced CT scan of the abdomen is the test of choice for the evaluation of splenic injury and most frequently reported (in 60 percent of cases) [13]. The sensitivity and specificity of the CT scan exceeded $95 \%$ in several reports $[14,15]$.

Ultrasonography is used less frequently (in 10.9 percent). It is an excellent tool and should be considered in all patients with hemodynamic instability. It is also a part of the ATLS protocol of investigation. A few cases of angiography have been reported (1.8 percent).

Diagnostic laparoscopy has been used in 23.6 percent of patient. This is especially significant in an hemodynamically unstable patient that requires a surgical exploration rather than a CT scan.

In the literature reviewed, splenectomy was the most commonly performed (67.9 percent), followed by conservative treatment (28.30 percent). One publication reported spleen rupture treated with arterial embolisation [8].

\section{Conclusion}

Spleen rupture is a rare complication of the colonoscopy. Mild discomfort is common even hours after colonoscopy, but significant abdominal pain and hemodynamic instabil- 
ity following the intervention should warrant a high index of suspicion for splenic injury [16].This is especially true for patients with previous surgical interventions causing potential splenocolic adhesions and for patients with previous history of abdominal trauma.

The significant sign of referred pain to the left shoulder tip as a result of diaphragmatic irritation can be present in up to 88 percent of cases [12]. Although sensitive, it not very specific, as gas distension alone may cause this in 50 percent of patients post uncomplicated colonoscopy [17]. The presence of hemodynamic compromise however, makes it very specific for splenic injury.

CT scan is regarded as the best diagnostic modality and an appreciable help for choosing the best treatment option. The severity of injury will determine whether management should be non operative or splenectomy.

\section{Competing Interests}

The author(s) declare that they have no competing interests.

\section{Authors' Contributions}

DP initiated the case series. HM analyzed and interpreted the patient data as well writing the manuscript. DP and HVR were major contributors in writing the manuscript. All authors read and approved the final manuscript.

\section{References}

1. Wolff WI, Shinya H. Colonofiberoscopy. JAMA. 1971;217(11):1509-1512.

2. Mandel JS, Church TR, Bond JH, Ederer F, Geisser MS, Mongin SJ, Snover DC, et al. The effect of fecal occultblood screening on the incidence of colorectal cancer. $\mathrm{N}$ Engl J Med. 2000;343(22):1603-1607.

3. Macrae FA, Tan KG, Williams CB. Towards safer colonoscopy: a report on the complications of 5000 diagnostic or therapeutic colonoscopies. Gut. 1983;24(5):376-383.

4. Schwesinger WH, Levine BA, Ramos R. Complications in colonoscopy. Surg Gynecol Obstet. 1979;148(2):270281.
5. Tsoraides SS, Gupta SK, Estes NC. Splenic rupture after colonoscopy: case report and literature review. J Trauma. 2007;62(1):255-257.

6. Pothula A, Lampert J, Mazeh H, Eisenberg D, Shen HY. Splenic rupture as a complication of colonoscopy: report of a case. Surg Today. 2010;40(1):68-71.

7. Heath B, Rogers A, Taylor A, Lavergne J. Splenic rupture: an unusual complication of colonoscopy. Am J Gastroenterol. 1994;89(3):449-450.

8. Stein DF, Myaing M, Guillaume C. Splenic rupture after colonoscopy treated by splenic artery embolization. Gastrointest Endosc. 2002;55(7):946-948.

9. Ahmed A, Eller PM, Schiffman FJ. Splenic rupture: an unusual complication of colonoscopy. Am J Gastroenterol. 1997;92(7):1201-1204.

10. Moore EE, Cogbill TH, Malangoni MA, Jurkovich GJ, Champion HR, Gennarelli TA, McAninch JW, et al. Organ injury scaling, II: Pancreas, duodenum, small bowel, colon, and rectum. J Trauma. 1990;30(11):1427-1429.

11. Gores PF, Simso LA. Splenic injury during colonoscopy. Arch Surg. 1989;124(11):1342.

12. Saad A, Rex DK. Colonoscopy-induced splenic injury: report of 3 cases and literature review. Dig Dis Sci. 2008;53(4):892-898.

13. Delgado Millan MA, Deballon PO. Computed tomography, angiography, and endoscopic retrograde cholangiopancreatography in the nonoperative management of hepatic and splenic trauma. World J Surg. 2001;25(11):1397-1402.

14. Peitzman AB, Makaroun MS, Slasky BS, Ritter P. Prospective study of computed tomography in initial management of blunt abdominal trauma. J Trauma. 1986;26(7):585-592.

15. Buntain WL, Gould HR, Maull KI. Predictability of splenic salvage by computed tomography. J Trauma. 1988;28(1):24-34.

16. Sumanac K, Zealley I, Fox BM, Rawlinson J, Salena B, Marshall JK, Stevenson GW, et al. Minimizing postcolonoscopy abdominal pain by using $\mathrm{CO}(2)$ insufflation: a prospective, randomized, double blind, controlled trial evaluating a new commercially available $\mathrm{CO}(2)$ delivery system. Gastrointest Endosc. 2002;56(2):190-194.

17. Janes SE, Cowan IA, Dijkstra B. A life threatening complication after colonoscopy. BMJ. 2005;330(7496):889890. 